\title{
Direct Detection of Phonons
}

\author{
Using an updated superconducting particle detector, researchers can \\ directly measure from its quasiparticle signal the number of phonons that \\ hit the detector.
}

By Rachel Berkowitz

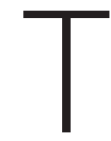
he bound pairs of electrons, known as Cooper pairs, that are responsible for carrying current in superconductors can unbind if they are hit with high-frequency phonons or photons. This unbinding is detrimental for superconductivity but has been leveraged to make single-particle detectors. Most of these detectors, however, require calibration or modeling for researchers to be able to infer from their signals the exact number of particles that hit the detector. Now, Elsa Mannila of Aalto University, Finland, and her colleagues have demonstrated a detector that can directly measure from an electrical current the number of broken Cooper pairs and thus particles that were absorbed [1].

The team fabricated their detector from aluminum, depositing a micrometer-sized piece of the material onto a silicon substrate. Next to the detector they deposited an aluminum "island," which they connected to a separate voltage source. When a current flowed across this island, it acted as a phonon emitter, generating high-frequency radiation that the team used to test the detector.

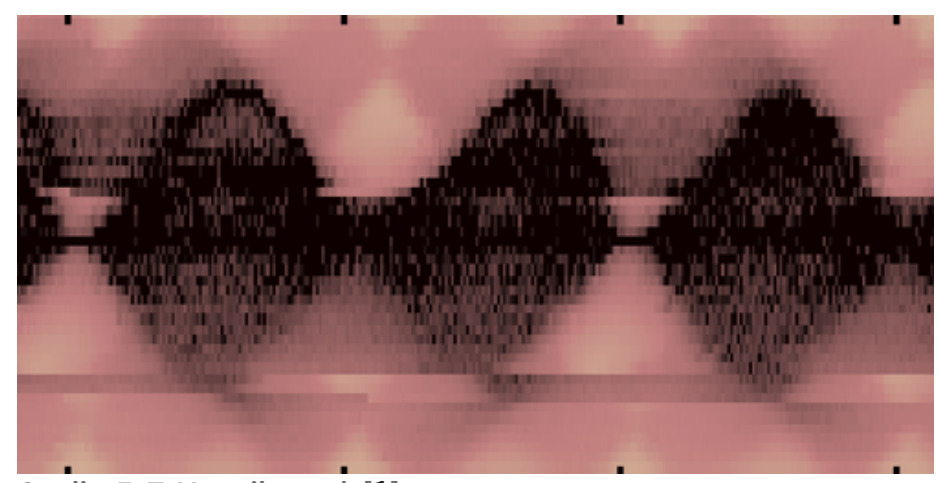

Credit: E. T. Mannila et al. [1]
Cooling the device to below $50 \mathrm{mK}$, where aluminum is a superconductor, the team found that the phonons from the emitter broke apart Cooper pairs in the detector when they hit it. The quasiparticles that were induced by that breaking apart (see Synopsis: Trapped Phonons Improve Photon Detection) escaped the detector, inducing in it a current as they left.

Comparing this current to the one used to drive the phonon emitter, the team found that the currents were directly proportional to one another, indicating that the pair-breaking rate was directly linked to the phonon generation rate. This dependency, which they found to be on-to-one, allowed the device to be used to directly measure the number of broken Cooper pairs without additional analyses. The researchers say that their device could be used to probe the presence and propagation of phonons in superconducting quantum systems.

Rachel Berkowitz is a Corresponding Editor for Physics based in Vancouver, Canada.

\section{REFERENCES}

1. E. T. Mannila et al., "Self-calibrating superconducting pair-breaking detector," Phys. Rev. Lett. 127, 147001 (2021). 